\title{
The Effect of a Functional Intensive Intervention Program on Self-Care in Children with Cerebral Palsy: A Case Study
}

\author{
Rosanne Roelofsma ${ }^{1^{*}}$ and Eugene Rameckers ${ }^{2,3,4}$ \\ ${ }^{1}$ Department of Health Sciences, FHML, Maastricht University, The Netherlands \\ ${ }^{2}$ Department of Functioning and Rehabilitation, CAPHRI, Maastricht University, The Netherlands \\ ${ }^{3}$ Center of Expertise, Adelante Rehabilitation Center, The Netherlands \\ ${ }^{4}$ Master of Specialized Physical Therapy, AVANS+, University of Applied Sciences, The Netherlands
}

*Corresponding author: Rosanne Roelofsma, Department of Health Sciences, FHML, Maastricht University, The Netherlands, E-mail: r.roelofsma@student.maastrichtuniversity.nl

\section{Abstract}

Former studies have shown that usual care in children with Cerebral Palsy (CP) often lacks effectiveness. Task-oriented functional intensive therapies have been shown to be promising for $\mathrm{CP}$ patients. The present case study aimed to examine the effect of a task-oriented functional intensive therapy program, so called FitCare $4 \mathrm{U}$, on the self-care of a 15-year-old male CP patient.

The subject in this case study was provided with 6 to 7 hours of therapy on a daily base adding up to a total of 70 hours of therapy in 15 days. Daily, one hour of this therapy was specific and aimed at the subject's personal goals. The remaining hours of therapy were based on physical motor activities to improve the subject's ability to perform daily activities, and were not specifically aimed at the subject's goals but on daily life activities. Motor activity tests and questionnaires were executed to assess the subject's functioning. These tests were structured in the domains of participation, activities and body function and structure of the International Classification of Functioning Disability and Health in Children and Youth (ICFCY). Pre-measurements took place 15 days before the intervention, post-measurements were executed on the final day of the intervention and follow-up measurements were carried out five months after the intervention. Next to the quantitative data obtained by the tests, qualitative data were obtained from a daily logbook.

The quantitative data suggest an improved ability to take part in daily situations and a decline in difficulties that the subject experiences in executing activities. The qualitative data suggest improvements in these areas and in the subject's body function \& structure.

Overall, this study suggests that the FitCare $4 \mathrm{U}$ program improved the self-care in this subject specifically in the ICFCY domains of participation and activities in both short and long term.
\end{abstract}

\author{
Keywords \\ Cerebral palsy, Functional intensive therapy, ICF-CY, Self- \\ Care
}

\section{Introduction}

The term Cerebral Palsy (CP) describes a group of permanent disorders of the development of movement and posture that are attributed to non-progressive disturbances that occurred in the developing fetal or infant brain, causing activity limitation [1]. The motor disorders of CP are often accompanied by disturbances of sensation, perception, cognition, communication, behavior, epilepsy and secondary musculoskeletal problems [1]. This group of diseases occurs in 1.4 to 4.0 per 1000 live births [2]. The activity limitations caused by CP are defined based on the International Classification of Functioning Disability and Health in Children and Youth (ICF-CY) [3].

Children with $\mathrm{CP}$ experience various problems during their development, as a result of the activity limitations they come across. Therapy is of great importance to make this development as successful as possible [3]. However, Novak, et al. claimed that outdated care is being provided to children with CP, with 10 to $40 \%$ of $\mathrm{CP}$ patients not receiving care that is based on recent scientific findings [4]. Next to this, Novak, et al. suggested that about $20 \%$ of therapies that are being given are unnecessary and even potentially dangerous [4]. This indicates that there is room for improvement in therapies for CP patients [4].

Citation: Roelofsma R, Rameckers E (2017) The Effect of a Functional Intensive Intervention Program on SelfCare in Children with Cerebral Palsy: A Case Study. Int J Brain Disord Treat 3:021. doi.org/10.23937/24695866/1410021

Received: September 20, 2017: Accepted: November 27, 2017: Published: November 29, 2017

Copyright: (C) 2017 Roelofsma R, et al. This is an open-access article distributed under the terms of the Creative Commons Attribution License, which permits unrestricted use, distribution, and reproduction in any medium, provided the original author and source are credited. 
Gordon, et al. showed that constraint-induced movement therapy and bimanual therapy result in improvements in hand function [5]. Constraint-induced movement therapy uses intensive unimanual practice along with restraint of the less-affected hand. Bimanual therapy maintains the intensity of practice that is applied in constraint-induced movement therapy, but both hands are involved in the training instead of only the most affected hand [5]. Furthermore, bimanual therapy in which not only the upper extremities but also the lower extremities are trained in a short and intensive functional training program was shown to significantly improve the functioning of children with bilateral CP [6]. Besides, Sorsdahl, et al. showed improved basic motor abilities in children with CP after a three-week period of intensive, goal-directed, activity-focused physiotherapy in a group setting [7]. Similar results were observed in the study of Young Hong, et al. in which it was shown that Gross Motor Function increased after eight weeks of intensive therapy [8]. This recent evidence points towards short and intensive functional therapies being very promising for CP patients [9].

The Dutch Association of Rehabilitation Physicians (2006) has drafted several criteria which therapies need to fulfill to be called functional intensive therapy [3]. First of all, the intervention has to be goal oriented, targeting the domains of activity and participation of the ICF-CY model. Furthermore, it should be task-and context-specific. To achieve this specificity, the needs of the child should form the starting point of the therapy. In addition, children and their parents need to be involved in finding solutions for the child's problems. Lastly, the therapy should not be aimed at normality, but should target functionality [3].

The Adelante Rehabilitation Centre in Valkenburg (the Netherlands) developed several treatment modalities to realize this task-oriented therapy in short and intensive methods. One of these treatments has been developed specifically for children with unilateral or bilateral CP in the Gross Motor Function Classification System (GMFCS) levels II, III and IV, and is called FitCare4U (Functional Intensive Therapy) [10].

FitCare4U is a short and intensive task-oriented therapy program designed for children between 12 and 18 -years-old with a physical disability. This therapy program implements the guidelines for task-oriented training in practice. Children with specific needs in the field of self-care are eligible for participation in the FitCare4U program. The goal of this program is to improve the subjects' self-care during a 15-day intervention. A therapy schedule is set up for the participants individually to warrant the specificity that is required for task-oriented training. The participant's individual goals and needs form the starting point of the therapy.

The present study examined the effect of the 15-day FitCare4U intervention program on the self-care of a 15-year-old boy diagnosed with bilateral spastic cerebral palsy. It is hypothesized that the FitCare4U program will improve the subject's self-care.

\section{Patient information}

The subject in this case study was a 15-year-old boy with a length of 1.64 meters and a weight of $62.2 \mathrm{~kg}$. The subject was born with bilateral spastic $\mathrm{CP}$, in which the left side of his body is more affected than the right side. He has GMFCS level II, which means that he experiences difficulties in walking under some circumstances, but walks in most settings [11].

Furthermore, he has MACS level III because he handles objects with difficulty and needs help to prepare and/or modify manual activities [12]. The subject also suffers from epilepsy, which is one of the accompanying impairments that may co-occur with CP [1]. Due to the severity of his epilepsy, which was life-threatening, it was necessary to perform a hemispherectomy in 2012. After the hemispherectomy, a craniotomy was needed. However, this resulted in worsening of the hemiparesis in the left body side.

The subject experienced most problems in the ICF$\mathrm{CY}$ domains of activities and participation. As regards the domain of activities, the subject mainly experienced difficulties in performing activities that require fine motor control and strength, especially if involvement of the left hand was necessary. The subject rarely used his left hand and arm, and neglected it most of the time because he was unable to use it properly. This neglection may have been worsened by the subject's lack of a complete field of view, which was an undesirable result of the hemispherectomy. Another consequence of the hemispherectomy was that the left hemisphere has taken over some of the functions of the non-functioning right hemisphere. As a result, the subject was unable to move his left and right arm and hand independently. These activity limitations together with the subject's low cognitive level and physical condition complicated his involvement in life situations, and thereby limited him at the level of participation.

The problems that the subject encountered were mostly related to the incomplete functioning of his left body side. This was expressed in the goals that he formulated based on his needs. These were: 1 ) Learning to tie his shoelaces; 2 ) Closing the buttons of his jeans; 3 ) Putting butter on a sandwich and cutting it, and 4) Doing his hair.

\section{Methods}

\section{Timeline}

Baseline measurements were taken fourteen days before the start of the intervention. Additionally, both the subject and his parents signed the informed consent form on this day. After these measurements, the subject continued his life for two weeks without any 


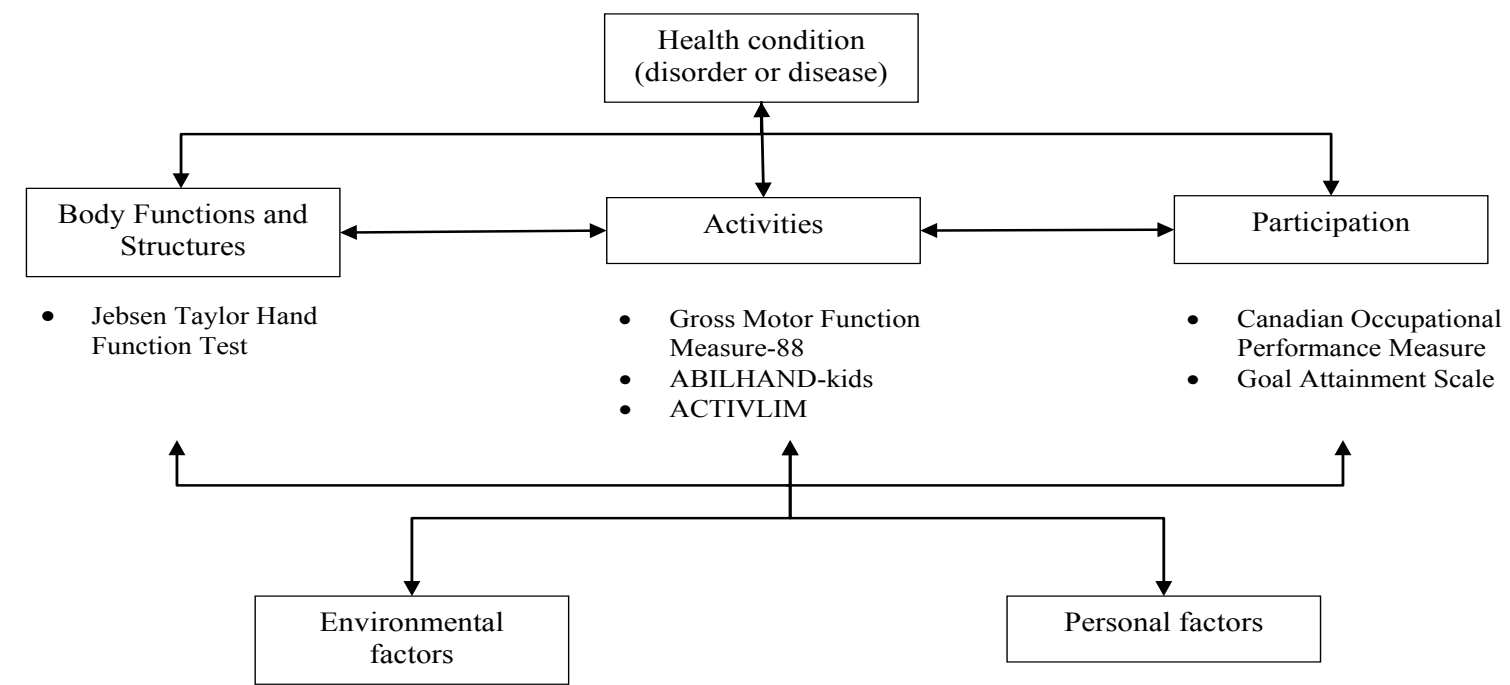

Figure 1: Overview of the tests executed in the different domains of the ICF-CY.

adjustments when compared to before the baseline measurements, where after he participated in the intervention program for 10 days uninterruptedly. After these 10 days, the subject went home for a 2-day because of Easter holidays after which he returned to the program for another 5 days. Post-measurements were assessed on the final day of the intervention. Follow-up measurements took place five months after the end of the intervention.

\section{Outcome measures}

Based on the subject's COPM goals, several tests have been executed to evaluate the different aspects of these goals. These goals were formulated at the ICF-CY level of participation and were based on his needs. To evaluate these goals properly, it was important to execute tests not only in the ICF-CY domain of participation, but also in the domains of activities and body function $\&$ structure. This is because there may be underlying problems in domains other than that of participation that influence the subject's ability to perform his goals. Therefore, it was necessary to map these three interconnected domains to find out the real problems that limited the subject in performing his goals.

As the subject formulated four goals that were all related to the intensive use of two hands, several hand function tests were performed to measure the intervention effect on hand function. The Canadian Occupational Performance Measure (COPM) was filled out for all of the subject's goals. A Goal Attainment Scale (GAS) was set up additionally for the primary COPM goal (learning to tie his shoelaces) to further evaluate his progress in performing this activity. Both the COPM and the GAS were executed to evaluate the subject's hand function on the level of participation. On the level of body function and structure, the Jebsen Taylor Hand function Test (JTHT) was carried out. On activity level, the ABILHAND-Kids and the ACTIVLIM were carried out. Because of the influence of gross motor function on daily life activities, and therefore also on the subject's goals, the Gross Motor Function Measure-88 (GMFM-88) was used as a standard to measure gross motor function. An overview of the tests executed in the different domains of the ICF-CY model is attached (Figure 1).

In addition to the quantitative data that were obtained from the executed tests, qualitative data were collected in a personal logbook. This was important to determine the subject's intervention adherence and tolerability. On a daily basis, the personal coach recorded relevant information about the subject's functioning on that day. For every activity during the day, data were collected concerning the time that the subject was actually active, and what this activity implied for the subject. Furthermore, a daily report about the individual therapy was written down in this logbook. Thereby, the progress that the subject made concerning his needs was kept.

\section{Tests to evaluate the ICF-CY domain of participation}

Canadian Occupational Performance Measure (COPM): The COPM is a measurement instrument that can be used to detect changes in the self-perception of the subject's performance of their needs and their satisfaction with this performance over time. The subject identifies problems that they experience in performing daily activities. For these activities, the subject their rates performance on a scale from 1 to 10 , in which 1 means they are not able to carry out this activity at all. If the subject is able to do this extremely well, they score a 10. Furthermore, the subject scores satisfaction with this performance, again on a 10-point scale with the same format as the performance scale [13]. The COPM was shown to have a good reliability and validity (ICC $=0.75$ ) [14]. In this case study, the subject identified his needs together with his parents. These needs were converted to the subject's personal goals, which formed the starting point for the therapy. The subject scored his performance and satisfaction on these needs two weeks before the intervention, and on the final day of 
the intervention.

Goal Attainment Scale (GAS): To evaluate the subject's progress in performing certain activities, a GAS can be constructed before treatment. For the subject in this case study, a GAS was set up for the primary COPM goal of tying his shoelaces (Appendix 1). First of all, a plan was constructed based on the subject's expected level of performance. After the treatment, the level of performance was scored on this scale. A score of -2 indicates the starting position. If the subject would score -3 , this indicates a decline. A score of -1 means that the subject did not fully accomplish his goal, whereas a score of 0 points out that the subject did accomplish his goal. If the subject achieved more than his goal, the score 1 will be given. If far more than the goal was achieved, the subject will score a 2 [15]. The GAS was shown to be sensitive to change, indicating a high responsiveness [16]. Only one study has investigated the validity of this scale, which found it to be acceptable [16]. However, there is insufficient knowledge about the reliability of the GAS when used in children. The score for the GAS was determined on the final day of the intervention.

\section{Test to evaluate the ICF-CY domain of body function}

\section{\& structure}

Jebsen Taylor Hand function Test (JTHT): The JTHT can be used as a tool to evaluate hand function. In this test, the subject was asked to perform 7 tasks which measure the hand activity that is used in daily life situations. These tasks were writing a short sentence (task 1 ), turning over cards (task 2), picking up small objects and placing them in a container (task 3 ), simulating eating (task 4), stacking checkers (task 5), moving empty large cans (task 6) and moving weighted large cans (task 7). However, the first task was excluded for the subject in this case study. Tasks 2 to 7 were performed with both the dominant hand (right) and the non-dominant hand (left). It was registered how long the subject needed to fulfill the task for each task separately. The maximum score of 120 seconds was assigned if the subject was not able to perform the task [17]. The JTHT was shown to be reliable $(r>0.60)$. However, there is no consensus concerning the validity of this test. Additionally, it was found that the JTHT has a poor responsiveness [18].

\section{Tests to evaluate the ICF-CY domain of activities}

ABILHAND-kids: The ABILHAND-kids questionnaire can be used to evaluate manual ability in children with $\mathrm{CP}$. In this questionnaire, 21 items that are representative for manual activities are included. Parents need to indicate whether fulfillment of the activities described by the 21 items is impossible, difficult or easy for their child. A question mark can also be filled out if the child has not attempted to carry out the activity in the last 3 months. However, if the activity was avoided due to an inability of performing it, the parents need to indicate that it is impossible for their child to carry out this ac- tivity. The ABILHAND-kids questionnaire was shown to have an excellent reliability with a Pearson's correlation coefficient of 0.94 [19]. This questionnaire was filled out by the subject's parents two weeks before the intervention and on the final day of the program. The results of the ABILHAND-kids were analyzed via the website of the Université de Louvain Catholique [20].

ACTIVLIM: The ACTIVLIM questionnaire is a measure of activity limitations in children and grown-ups diagnosed with a neuromuscular disease, and can therefore also be used in children with CP. Parents need to indicate whether it is impossible, difficult or easy for their child to perform each of the 22 activities included in this questionnaire. These 22 items are all representative for activities of daily living. If the child has not performed the activity in the prior 3 months, a question mark needs to be filled out. However, if the activity was avoided due to an inability of performing it, the parents need to indicate that it is impossible for their child to carry out this activity. The ACTIVLIM questionnaire was shown to be reliable with an intra-class correlation coefficient of 0.96 [21]. This questionnaire was filled out by the subject's parents two weeks before the intervention and on the final day of the program. Similar to the ABILHAND-kids, the results of the ACTIVLIM were analyzed via the website of the Université de Louvain Catholique [20].

Gross Motor Function Measure-88 (GMFM-88): The GMFM-88 enables the evaluation of changes in gross motor function in children with CP. This measurement instrument consists of 88 items concerning gross motor function, divided into four different domains. Domain A contains items concerning lying and rolling over. In domain $B$, items about sitting are included. Domain C consists of items concerning crawling and kneeling. Domain $\mathrm{D}$ is focused on standing and domain $\mathrm{E}$ contains items about walking, running and jumping. Four different scores can be awarded. If a child does not try to perform the activity, they will score a 0 . Children who try but do not succeed will score a 1 . The score 2 is granted if the child carries out the task partially. Children who fulfill the task completely will score a 3 [22]. The GMFM-88 was shown to have an excellent reliability (ICC $=0.95$ ) and a reasonable responsiveness (no numbers given) [23]. This test was performed two weeks before the start of the intervention and on the last day of the program. The subject was wearing regular sportswear and his ankle-foot orthosis (without shoes) during this test.

\section{Therapeutic intervention}

The subject participated in the FitCare4U intervention program together with seven other youngsters with other diagnoses (e.g. spina bifida, stroke and juvenile Parkinson), who all had their own personal coaches. The subject in this case study was guided by one Health Sciences student and one physical therapy student. 
These students were in turn guided by an experienced occupational therapist.

The time schedule was in general comparable for most days of the intervention. On weekdays, the participant attended school in the morning. He was picked up from school and there was a joint lunch with all participants and supervisors. Most of the days, lunch was followed by an hour of individual therapy, focused on the subject's personal goals. If this was not the case, the individual therapy was implemented in the program at another moment during the day. This specified, goal-oriented therapy was important for improving the subject's needs. The remaining hours of therapy were based on physical motor activities to improve the subject's ability to perform daily activities, and were not specifically aimed at the subject's goals but on daily life activities. Still, the subject's goals were involved in the activities as much as possible. Examples of the activity-based therapy in this intervention program were circus and decoration activities, dinner preparations and the group activities planned in the afternoon. The activity based therapy was generally planned after the goal-oriented therapy and differed every day. This was always challenging and demanding for the subject since intensive physical activity was required during all activities. After the activity based therapy, the participants and interventionists had dinner together. In the evening, relaxing activities such as playing board games were planned before the participants went to bed. During the weekend, the program started at $9.00 \mathrm{~h}$ since there was no school. The same components were implemented in the program as during weekdays, only in a different order. After breakfast, the individual therapy took place. The time until lunch was filled up with activity-based therapy, similar to the time from lunch until dinner. In the evening, relaxing activities were planned before the participants went to bed.

During the program, the subject received six to seven hours of therapy on a daily basis (one hour of individual, goal-oriented therapy, the remaining hours were spent on activity-based therapy). In total, the subject received 70 hours of therapy within 15 days. The program for one ordinary weekday and one weekend day is attached (Appendix 2).

This intervention program was aimed at improving several domains of the ICF-CY model, and thereby facilitating the subject in performing his needs. The domain of activities was integrated in this program by training daily life skills during the activity-based therapy in the afternoon. To improve body function and structure, there was a focus on an active attitude of the participant all day long. For example, the subject could only sit on stools without backrest. To make his sitting position even more active, all stools were provided with a balance cushion. Sitting on these cushions constantly requires small movements to keep balance. Furthermore, usage of walking tools was avoided, and was only allowed if necessary. Thus, the participant was constantly pushed to function at his maximal capacity.

For the subject in this case study, the focus of the FitCare4U intervention was mainly on improving his left arm and hand function, which was important for his formulated needs. Furthermore, he was stimulated to use his affected arm and hand more frequently to improve motor control and strength.

\section{Needs directed intervention}

The subject's needs described in the patient information were formulated as COPM goals to be able to evaluate them quantitatively using the COPM.

The subject's first COPM goal was learning to tie his shoelaces. This was practiced in every therapy session. The first step in this process was to teach the subject the steps involved in tying his shoelaces. After the participant remembered the steps, he practiced them on a shoe that was standing on the table. It took the participant seven therapy sessions to get used to doing this. After the subject became familiar with this activity, he put on his shoes and practiced tying the shoelaces while wearing his shoes. Since the participant was not able to fully involve his left hand, it was necessary to look for a method that does not require constant involvement of both hands. After five days, the subject's coaches found such a method that enabled the subject to tie his shoelaces.

The second COPM goal was being able to close the buttons of his jeans completely independently. Practicing this was involved in every individual therapy session. First, the participant practiced closing the buttons of jeans that were lying on a table. Once he controlled this movement, he practiced to close the buttons while wearing his jeans.

The third COPM goal was putting butter on a sandwich and cutting it. This was only practiced during lunch every day, not during the individual therapy sessions.

The fourth COPM goal for the subject was doing his hair. This activity was involved in about half of the individual therapy sessions.

\section{Results}

\section{Results in the ICF-CY domain of participation}

The subject showed progress in all COPM goals ( $\mathrm{Ta}$ ble 1). During the post-measurement, the greatest improvements were seen in tying his shoelaces (goal 1 ), putting butter on a sandwich and cutting it (goal 3 ) and doing his hair (goal 4). The subject was not able to perform these activities before the intervention. However, the subject was able to carry out these activities in a reasonable way with which he was rather satisfied directly after the intervention. The subject also gave a higher score for both the execution of and his satisfaction with 
Table 1: Results of the pre- and post-measurements of the executed tests in the domain of participation.

\begin{tabular}{|l|l|l|l|l|}
\hline Test & & Pre-measurement & Post-measurement & Follow-up \\
\hline $\begin{array}{l}\text { COPM } \\
\text { (score) }\end{array}$ & Goal 1 & $\mathrm{P}=1$ & $\mathrm{P}=6$ & $\mathrm{P}=8$ \\
& (Tying shoelaces) & $\mathrm{S}=1$ & $\mathrm{~S}=7$ & $\mathrm{~S}=9$ \\
& Goal 2 & $\mathrm{P}=6$ & $\mathrm{P}=7$ & $\mathrm{P}=7$ \\
& (Closing jeans buttons) & $\mathrm{S}=6$ & $\mathrm{~S}=7$ & $\mathrm{~S}=6$ \\
& Goal 3 & $\mathrm{P}=1$ & $\mathrm{P}=6$ & $\mathrm{P}=8$ \\
& (Putting butter on a sandwich and cutting it) & $\mathrm{S}=1$ & $\mathrm{~S}=7$ & $\mathrm{~S}=8$ \\
& Goal 4 & $\mathrm{P}=1$ & $\mathrm{P}=7$ & $\mathrm{P}=8$ \\
& (Doing hair) & $\mathrm{S}=1$ & $\mathrm{~S}=7$ & $\mathrm{~S}=9$ \\
\hline Goal Attainment Scale (score) & -2 & 2 & 2 \\
\hline
\end{tabular}

COPM: Canadian Occupational Performance Measure; P: Performance; S: Satisfaction.

Table 2: Results of the pre- and post-measurements of the executed test in the domain of body function \& structure.

\begin{tabular}{|c|c|c|c|c|}
\hline \multirow{3}{*}{$\begin{array}{l}\text { Test } \\
\text { Jebsen Taylor } \\
\text { Test (sec) }\end{array}$} & \multirow[b]{2}{*}{ Task 1 - Writing a short sentence } & \multirow{2}{*}{$\begin{array}{l}\text { Pre-measurement } \\
\text { Not executed }\end{array}$} & \multirow{2}{*}{$\begin{array}{l}\text { Post-measurement } \\
\text { Not executed }\end{array}$} & \multirow{2}{*}{$\begin{array}{l}\text { Follow-up } \\
\text { Not executed }\end{array}$} \\
\hline & & & & \\
\hline & Task 2 - Turning over cards & $D=5.58$ & $\mathrm{D}=3.66$ & $D=5.28$ \\
\hline & & $N=28.65$ & $N=31.06$ & $N=40.06$ \\
\hline & Task 3 - Putting objects in a container & $D=9.68$ & $D=7.69$ & $D=10.08$ \\
\hline & & $N=48.98$ & $N=47.79$ & $N=102.61$ \\
\hline & Task 4 - Simulate eating & $D=17.65$ & $D=11.15$ & $D=14.38$ \\
\hline & & $N=120$ & $N=120$ & $N=120$ \\
\hline & Task 5 - Stacking checkers & $D=4.90$ & $D=4.60$ & $D=3.77$ \\
\hline & & $N=39.97$ & $N=37.14$ & $N=40.83$ \\
\hline & Task 6 - Moving empty large cans & $D=4.30$ & $D=2.93$ & $D=4.05$ \\
\hline & & $N=22.65$ & $N=34.01$ & $N=33.76$ \\
\hline & Task 7 - Moving weighted large cans & $D=4.24$ & $D=3.42$ & $D=4.87$ \\
\hline & & $N=59.14$ & $N=120$ & $N=28.68$ \\
\hline & Total & $D=46.35$ & $D=33.45$ & $D=42.43$ \\
\hline & & $N=319.39$ & $N=390$ & $N=365.94$ \\
\hline
\end{tabular}

Sec: Seconds; D: Dominant hand; N: Non-dominant hand.

closing the buttons of his jeans (goal 2). However, the progress for this goal was not as great as for goals 1 , 3 and 4 (Table 1). During the follow-up measurement, the subject's performance and satisfaction with goals 1, 3 and 4 increased even more when compared to the post-measurement. His performance of goal 2 was the same as during the post-measurement and his satisfaction with this performance on this goal decreased slightly (Table 1 ). The results of the GAS suggest that the subject made a lot of progress concerning the first COPM goal of tying his shoelaces, since it increased from -2 to maximum improvement (score 2) during the intervention. Before the start of the intervention, the subject was only able to put on his shoes and draw the laces so that the shoe sat tightly around the foot. However, after the intervention he was able to tie his shoelaces completely independently. With this achievement, the subject accomplished much more than the goal. The follow-up measurement indicates a complete preservation of the effect five months after the intervention (Appendix 1 and Table 1).

\section{Results in the ICF-CY domain of body function \& structure}

An improvement of 12.90 seconds was observed between the pre- and post-measurements of the JTHT for the dominant (right) hand. The subject needed 70.61 seconds more to perform all activities with his non-dominant (left) hand during the post-measurement when compared to the pre-measurement (Table 2). During the follow-up measurement, it took the subject 8.98 seconds more to fulfill all tasks with his dominant hand when compared to the post-measurement. However, he still needed less time to do so than during the pre-measurement. When comparing the results for the non-dominant hand during the follow-up measurement to the results of the pre-measurement, 46.55 seconds more were needed for the subject to perform all activities Still, the follow-up results are better than the post-measurement results, as the subject needed 24.06 seconds less to complete all tasks during the follow-up measurement when compared to the post-measurement (Table 2).

\section{Results in the ICF-CY domain of activities}

All tests that were performed to evaluate the subject's functioning in the ICF-CY domain of activities showed better results for the post-measurements when compared to the pre-measurements (Table 3). The subject achieved increased log it scores in the ABILHAND-kids and ACTIVLIM tests during the post-measurements in comparison to the pre-measurements. The improvement measured during the post-measure- 
Table 3: Results of the pre- and post-measurements of the executed tests in the domain of activities.

\begin{tabular}{|l|l|l|l|}
\hline Test & Pre-measurement & Post-measurement & Follow-up \\
\hline ABILHAND-Kids (logit) & 1.203 & 3.900 & 2.172 \\
\hline ACTIVLIM (logit) & 1.475 & 4.032 & 4.032 \\
\hline Gross Motor Function Measure (percentage) & $95.8 \%$ & $98.1 \%$ & $98.0 \%$ \\
\hline
\end{tabular}

\section{Gross Motor Function Measure}

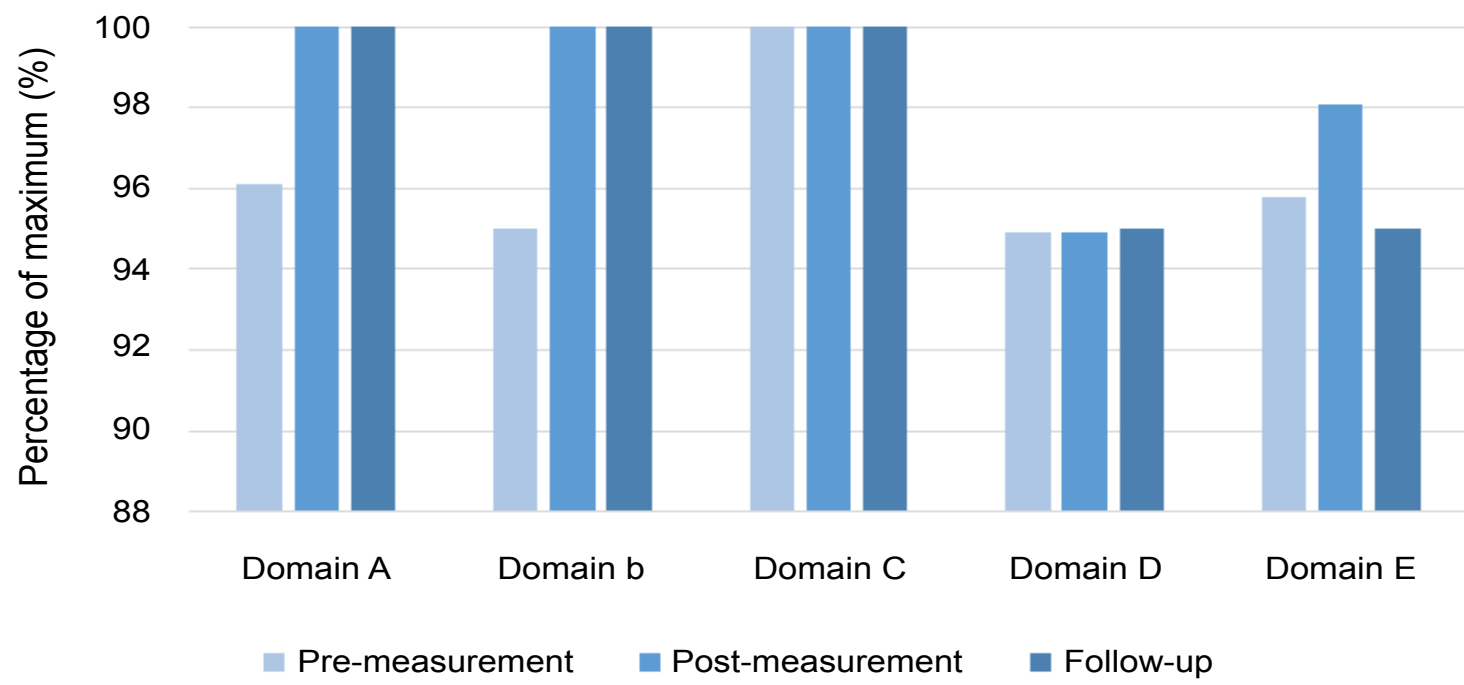

Figure 2: The subject's performance in the separate domains of the Gross Motor Function Measure, and his total performance in per cent. Domain $A=$ lying and rolling over, domain $B=$ sitting, domain $C=$ crawling and kneeling, domain $D=$ standing, domain $E=$ walking, running and jumping.

ment in the ACTIVLIM was fully preserved throughout the 5-months follow-up. For the ABILHAND-kids, the log it scores decreased slightly during the follow-up measurement when compared to the post-measurement. However, it was still higher than during the pre-measurement (Table 3). As regards the GMFM-88, increased scores were visible in domains $A$ (lying and rolling over), B (sitting) and $\mathrm{E}$ (walking, running and jumping). The subject's performance in domains $C$ (crawling and kneeling) and $D$ (standing) did not differ between the pre- and post-measurements. In domain A, the subject's score rose from $96.1 \%$ during the pre-measurements to $100 \%$ during the post-measurements. An increase of $5 \%$ was seen in domain B. The subject scored $100 \%$ in domain $C$ and $94.9 \%$ in domain $D$ during both the pre-measurements and the post-measurements. In domain $\mathrm{E}$, the subject's performance increased from $95.8 \%$ to 98.1\% (Figure 2). The subject's total performance also increased (Table 3). The improvements in the domains $A, B, C$ and $D$ that were seen during the post-measurements were still present during the follow-up measurement. For domain $\mathrm{E}$, a small decline in score was seen during the follow-up measurement when compared to both the pre- and the post-measurement (Figure 2). The follow-up measurements show a preservation of the intervention effect on overall gross motor function five months after the intervention (Table 3 ).

\section{Results from the qualitative data in the logbook}

The qualitative data that were collected in the log- book indicate a great intervention adherence and tolerability for the subject in this case study. He was eager to achieve his personal goals and therefore had a motivated and cooperative attitude during the whole intervention. He participated enthusiastically in all activities and visibly enjoyed his participation in the program.

The subject's ability to perform his needs, described as COPM goals, showed improvements according to the qualitative data. The qualitative data specifically showed great progress for the first COPM goal of tying his shoelaces. Although the subject was not able to tie his shoelaces at the beginning of the intervention and did not know the steps involved in this activity, he was able to tie his shoelaces independently on the eleventh day of the intervention. The qualitative data also indicate great improvements in the subject's second COPM goal of learning to close the buttons of his jeans independently. At the start of the intervention, he was not able to do this without any help. However, he could do this independently on the sixth day of the intervention according to the qualitative data. Similarly, the qualitative data concerning the third and fourth COPM goals (putting butter on a sandwich and cutting it, and doing his hair) indicate great improvements for the subject to perform these activities.

\section{Discussion}

Summarizing the results, improved test scores were specifically observed in the ICF-CY domains of partici- 
pation and activities for the subject in this case study. Improvements were less clear in the ICF-CY domain of body function and structure. Nevertheless, the qualitative data indicate progress in both the ICF-CY domains of participation and activities, and in the domain of body function and structure. Overall, these results suggest that the FitCare $4 U$ program improved the self-care for the subject in this case study.

Two mechanisms are expected to underlie the changes observed in this case study. First of all, the neuroplastic changes induced by intensive motor learning interventions may have contributed to the observed improvements in the domains of participation and activities [6]. Secondly, the increased strength that results from intensive therapy was shown to be beneficial for functional abilities in children with bilateral CP in previous studies, and is therefore expected to contribute to the changes in this case study as well [6].

The results obtained from the tests in the ICF-CY domain of participation clearly indicate progress. The results of the GAS point towards improvements as regards the subject's ability to tie his shoelaces, which corresponds to the results of the COPM for this goal. In addition, the quantitative data in this domain generally correspond to the qualitative data that were based on observations. However, the qualitative data suggest great improvements for all COPM goals, whereas the data obtained from the COPM test indicate only small improvements for the subject's ability to close the buttons of his. The COPM test was filled out by the subject's parents, thus the quantitative data were obtained from the parents. However, the qualitative data in the logbook were obtained from the subject's coaches. This difference in assessors might be the declaration for the discrepancy in the data. Still, all of the results suggest improvements in the subject's goals. This is in accordance with the findings in the study of Bleyenheuft, et al. In this study, the effect of hand-arm bimanual intensive therapy including lower extremity in children with bilateral CP was examined in a quasi-randomized trial [6]. This study is a good comparison with the study of Bleyenheuft, as the intensity and duration of the therapy were roughly the same (84 hours of therapy in 13 days in the study of Bleyenheuft, vs. 70 hours of therapy in 15 days in this case study). Moreover, the effects of the therapy were studied in children with bilateral $C P$ (GMFCS II) in both studies. In the study of Bleyenheuft, it was shown that hand-arm bimanual intensive therapy including lower extremity significantly improved COPM scores in the treatment group, but not in the control group [6]. Improved scores were observed for both the execution of the goals and the satisfaction with this, which was also observed in this case study.

The results obtained from the tests in the ICF-CY domain of body function \& structure generally do not indicate progress. However, improvements were expected in this domain based on the results observed in the study of Bleyenheuft, et al. [6]. The results of the JTHT indicate a decline in the subject's non-dominant hand function. On the contrary, the results of the COPM and the qualitative data suggest improvements in his non-dominant hand function. The observed decline shown by the JTHT was to a great extent caused by the test results of the seventh item of this test (moving weighted large cans) during the post-measurements. The subject could not perform this task during the post-measurements, although he could perform it during the pre-measurements. During the follow-up measurements, the subject needed much more time to perform task 3 (putting small objects in a container) when compared to the pre-measurements. The total time that the subject needed to perform all tasks during the post-measurements and follow-up increased mainly because of his lacking ability to perform these tasks.

The results obtained from the tests in the domain of activities clearly indicate progress, as improved scores were seen for all tests executed in this domain. This is in accordance with the studies of Novak, et al., Sorsdahl, et al. and Bleyenheuft, et al. in which improved motor activity was shown as a result of goal-oriented training $[6,7,24]$.

Previous research into the efficacy of short and intensive functional therapy only examined the intervention effect on hand and arm function, sometimes including leg function and gross motor function as well. However, the complexity of the problems goes far beyond this, as the subject's self-care also plays an important role. The effect of short and intensive functional therapies on self-care has not been investigated before. The FitCare4U intervention examines the effect of a short and intensive functional therapy program on the participant's self-care, by only including children with specific needs in the field of self-care. For the subject in this case study, all of his needs were important elements for his self-care. This emphasis on self-care makes this case study innovative and unique. How the therapy is given makes the FitCare4U program unique as well. The intensity and duration of the therapy that was shown to be effective in previous studies is maintained in the FitCare4U program, but it is given in a way that is fun and motivating for the participants as well by implementing therapy in group activities that the participants enjoy doing.

This case study has several constraints. First of all, the timing of the post-measurements was not ideal, since the subject was exhausted on the day that the post-measurements took place. This specifically influenced the results in the ICF-CY domain of body function \& structure. Based on previous studies, progress might have been seen in this domain as well if these tests were performed when the subject was well rested $[6,24]$. Therefore, it might be better to perform some of 
the tests later. However, performing tests later in time will complicate the researcher in evaluating the shortterm effects of the FitCare4U intervention, so this also has its drawbacks. Besides, a case study only describes the results of one subject in detail and therefore has a very low generalizability. A larger population is needed to be able to draw a conclusion about the efficacy of the FitCare4U intervention in general. However, it is still important to analyze the effect of the FitCare4U intervention on one subject elaborately. A case-study enables the researcher to detect the intervention effects on the subject very detailed. This is not possible in other studies with more statistical power, in which mean scores are analyzed. Since no evidence is provided for the effect of functional intensive therapy programs on self-care yet, it is important to analyze this in a case study first. This case study can now underlie the results of studies with more power, to investigate the efficacy of the FitCare4U intervention program on self-care in other children with CP.

In short, this case study indicates that the FitCare4U program improved the subject's functioning specifically in the ICF-CY domains of participation and activity, thereby improving his self-care. This suggests that this program could possibly also have beneficial effects in other youngsters with bilateral spastic cerebral palsy. Further research in studies with a larger sample size and a control group included are needed to confirm this.

\section{References}

1. Rosenbaum $P$, Paneth $N$, Leviton A, Goldstein M, Bax M, et al. (2007) A report: The definition and classification of cerebral palsy April 2006. Dev Med Child Neurol Suppl 109: 8-14.

2. Tulchinsky TH, Varavikova EA (2014) Special Community Health Needs. In: The New Public Health. ( $3^{\text {rd }}$ edn), ACADEMIC PRESS, 381-418.

3. Nederlandse Vereniging van Revalidatieartsen (2015) Richtlijn Diagnostiek en Behandeling van kinderen met spastische Cerebrale Parese. Utrecht.

4. Novak I (2014) Evidence-based diagnosis, health care, and rehabilitation for children with cerebral palsy. J Child Neurol 29: 1141-1156.

5. Gordon AM, Hung YC, Brandao M, Ferre CL, Kuo HC, et al. (2011) Bimanual training and constraint-induced movement therapy in children with hemiplegic cerebral palsy: A randomized trial. Neurorehabil Neural Repair 25: 692-702.

6. Bleyenheuft $Y$, Ebner-Karestinos D, Surana B, Paradis J, Sidiropoulos A, et al. (2017) Intensive upper- and lower-extremity training for children with bilateral cerebral palsy: A quasi-randomized trial. Dev Med Child Neurol 59: 625-633.

7. Sorsdahl AB, Moe-Nilssen R, Kaale HK, Rieber J, Strand LI (2010) Change in basic motor abilities, quality of movement and everyday activities following intensive, goal-directed, activity-focused physiotherapy in a group setting for children with cerebral palsy. BMC Pediatr 10: 26.

8. Young Hong B, Jo L, Sung Kim J, Hoon Lim S, Min Bae $J$ (2017) Factors Influencing the gross motor outcome of intensive therapy in children with cerebral palsy and developmental delay. J Korean Med Sci 32: 873-879.

9. Sakzewski L, Ziviani J, Boyd RN (2014) Efficacy of upper limb therapies for unilateral cerebral palsy: A Meta-analysis. Pediatrics 133: e175-e204.

10. Adelante Rehabilitation Centre. FitCare4U. Netherlands.

11. Robert Palisano, Peter Rosenbaum, Doreen Bartlett ML (2007) Gross Motor Function Classification System Expanded and Revised. Cent Child Disabil Res 2: 4.

12. Eliasson AC, Krumlinde-Sundholm L, Rösblad B, Beckung E, Arner M, et al. (2006) The Manual Ability Classification System (MACS) for children with cerebral palsy: Scale development and evidence of validity and reliability. Dev Med Child Neurol 48: 549-554.

13. Mary Law, Sue Baptiste, Anne Carswell, Mary Ann McColl, Helene Polatajko, et al. (2000) Canadian Occupational Performance Measure. CAOT Publications ACE, 1-4.

14. Reid D, Campbell K (2006) The use of virtual reality with children with cerebral palsy: A pilot randomized trial. Therapeutic Recreation Journal 40: 255-268.

15. Kiresuk TJ, Sherman RE (1968) Goal attainment scaling: A general method for evaluating comprehensive community mental health programs. Community Ment Health $\mathrm{J} 4$ : 443-453.

16. Steenbeek D, Ketelaar M, Galama K, Gorter JW (2007) Goal attainment scaling in paediatric rehabilitation: A critical review of the literature. Dev Med Child Neurol 49: 550-556.

17. Jebsen RH, Taylor N, Trieschmann RB, Trotter MJ, Howard LA (1969) An objective and standardized test of hand function. Arch Phys Med Rehabil 50: 311-319.

18. Sears ED, Chung KC (2010) Validity and responsiveness of the Jebsen-Taylor Hand Function Test. J Hand Surg Am 35: 30-37.

19. Arnould C, Penta M, Renders A, Thonnard JL (2004) ABILHAND-Kids: A measure of manual ability in children with cerebral palsy. Neurology 63: 1045-1052.

20. (2017) Rehab Scales. Université Catholique de Louvain.

21. Vandervelde L, Van den Bergh PYK, Goemans N, Thonnard JL (2009) Activity limitations in patients with neuromuscular disorders: A responsiveness study of the ACTIVLIM questionnaire. Neuromuscul Disord 19: 99-103.

22. Maltais DB, Bar-Or O (2006) Assessing functional differences in gross motor skills in children with cerebral palsy who use an ambulatory aid or orthoses: Can the GMFM-88 help? Dev Med Child Neurol 48: 158-159.

23. Ko J, Kim M (2013) Reliability and responsiveness of the gross motor function measure-88 in children with cerebral palsy. Phys Ther 93: 393-400.

24. Novak I, Mcintyre S, Morgan C, Campbell L, Dark L, et al. (2013) A systematic review of interventions for children with cerebral palsy: State of the evidence. Dev Med Child Neurol 55: 885-910. 
Appendix 1: Goal Attainment Scale for tying the shoelaces.

\section{Setting}

\section{Definition}

The subject is sitting with shoes within reach. Putting on the shoes including tying the shoelaces consists of the following steps/qualities of execution:

1. Putting on shoes and draw laces so that the shoe sits tightly around the foot

2. Learning and remembering how shoelaces should be tied

3. Making a knot with the shoelaces (on a shoe that is standing on the table)

4. Making a loop with one shoelace (on a shoe that is standing on the table)

5. 'Catching' the loop with the other shoelace, while the loop is being held by someone else (on a shoe that is standing on the table)

6. Finishing the bow while the loop is being held by someone else (on a shoe that is standing on the table)

7. 'Catching' the loop with the other shoelace, while the loop is being held by the subject (on a shoe that is standing on the table)

8. Finishing the bow while the loop is being held by the subject (on a shoe that is standing on the table)

9. Making a knot with the shoelaces (while wearing the shoe)

10. Making a loop with one shoelace (while wearing the shoe)

11. 'Catching' the loop with the other shoelace, while the loop is being held by someone else (while wearing the shoe)

12. Finishing the bow while the loop is being held by someone else (while wearing the shoe)

13. 'Catching' the loop with the other shoelace, while the loop is being held by the subject (while wearing the shoe)

14. Finishing the bow while the loop is being held by the subject (while wearing the shoe)

Manner of measurement

\section{Assignment}

It is observed which of the described steps/qualities of execution are being fulfilled

\section{Goal attainment level}

-3 Decline

-2 Starting position

-1 Less than the goal

0 Goal

Put on your shoes and tie the shoelaces

1 More than the goal Child fulfills 9 to 12 of the first 12 described steps of execution

2 Much more than the Child fulfills 13 to 14 of the 14 described steps of execution goal

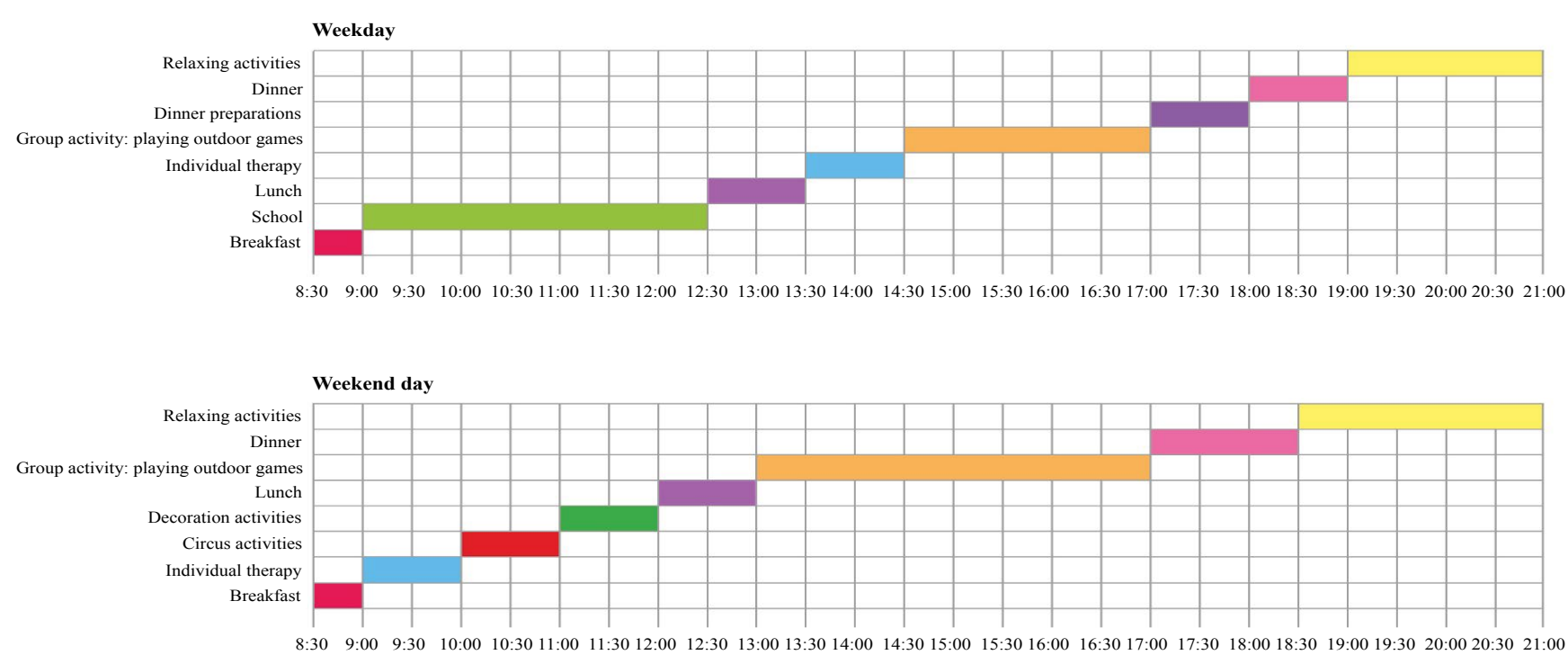

Appendix 2: Program for one ordinary weekday and one weekend day. 